\title{
Physicochemical sorption properties of geologic materials by inverse gas chromatography
}

\author{
E.H. DENIS*, N.L. HugGeTt, W.C. WeAVER,
} L.A. RuSH, C.G. FrAGA, A.J. CARMAN

Pacific Northwest National Laboratory, Richland, WA, USA

(*correspondence: elizabeth.denis@pnnl.gov)

To better understand how volatile compounds are transported through geologic materials, we are using inverse gas chromatography (IGC) to determine gas-solid sorption properties of a suite of materials. IGC allows us to characterize and quantify the physicochemical properties of particulates (e.g., soil and sand) of various mineralogical makeup and grain sizes. This is accomplished by measuring the level of interaction between the uncharacterized material and known probe gases by varying temperature, humidity, and carrier gas flow. By injecting a known probe gas through a packed sample column, sorption properties are determined based on peak shape and retention time of the gas relative to an unretained species (e.g., $\mathrm{CH}_{4}$ ). The probe gases can be surrogates for additional compounds of interest (e.g., volatile radionuclides and other environmental contaminants) and cover a wide-range of polarities and molecular structures. The results (e.g., diffusion and partition coefficients and heat of sorption) help to decipher sorption effects for geologic materials under different temperature and humidity conditions. Such results may help define boundary conditions for inputs in computational models to simulate gas transport.

The inherent physical and chemical heterogeneity of soil and many geologic materials can make prediction of sorption properties difficult. Characterizing the properties of individual organic and inorganic components can elucidate the primary factors influencing sorption interactions in more complex mixtures. Based on our collected heat of sorption values, soil and bentonite clay have greater sorption to nonpolar alkanes than simpler single mineral media (e.g., salt, quartz sand, and calcium carbonate). The interactivity of soils and clays are likely heightened by the variety of bonding sites in the complex structures compared to the single mineral media. For diffusion coefficient, we have observed a positive correlation with temperature and that there is less variability in results between samples of homogeneous sorbent material than heterogeneous soil. We discuss the capabilities and challenges of using IGC to characterize the physicochemical properties of geologic materials and inform gas transport. 